\title{
What is the role of the meson cloud in the $\Sigma^{* 0} \rightarrow \gamma \Lambda$ and $\Sigma^{*} \rightarrow \gamma \Sigma$ decays?
}

\author{
G. Ramalho ${ }^{1}$ and K. Tsushima ${ }^{1}$ \\ ${ }^{1}$ International Institute of Physics, Federal University of Rio Grande do Norte, \\ Avenida Odilon Gomes de Lima 1722, Capim Macio, Natal-RN 59078-400, Brazil
}

(Dated: June 2, 2021)

\begin{abstract}
We study the effect of the meson cloud dressing in the octet baryon to decuplet baryon electromagnetic transitions. Combining the valence quark contributions from the covariant spectator quark model with those of the meson cloud estimated based on the flavor SU(3) cloudy bag model, we calculate the transition magnetic form factors at $Q^{2}=0\left(Q^{2}=-q^{2}\right.$ and $q$ the four-momentum transfer), and also the decuplet baryon electromagnetic decay widths. The result for the $\gamma^{*} \Lambda \rightarrow \Sigma^{* 0}$ decay width is in complete agreement with the data, while that for the $\gamma^{*} \Sigma^{+} \rightarrow \Sigma^{*+}$ is underestimated by 1.4 standard deviations. This achievement may be regarded as a significant advance in the present theoretical situation.
\end{abstract}

\section{INTRODUCTION}

One of the most interesting challenges in hadronic physics is to study the internal structure of baryons and mesons. A microscopic understanding of the transition between the hadronic states is also very important. Although it is generally accepted that the internal structure of hadrons and the dynamics of quarks and gluons, are described by quantum chromodynamics (QCD), one has to rely on some effective degrees of freedom in the nonperturbative low $Q^{2}$ region such as constituent quarks which form baryon cores with meson cloud excitations [1, 2]. Although there exist some works which attempted to treat the meson cloud explicitly as the $q \bar{q}$ excitations in the so-called unquenched quark models [36], most of the phenomenological models treat the meson cloud using pointlike meson excitations.

Particular examples of very interesting studies may be the electromagnetic transitions between an octet baryon $B(\operatorname{spin} 1 / 2)$ and a decuplet baryon $B^{\prime}(\operatorname{spin} 3 / 2)$, $\gamma^{*} B \rightarrow B^{\prime}$, and the $B^{\prime}$ electromagnetic decay reactions, $B^{\prime} \rightarrow \gamma B$. There are theoretical predictions for the $\gamma^{*} B \rightarrow B^{\prime}$ transition magnetic moments based on quark models [7-13], including quark models with meson cloud dressing [14 17], Skyrme and soliton models [18, 19], large $N_{c}$ limit 20], QCD sum rules [21, 22], and chiral perturbation theory [23]. There are also some results from lattice QCD [24, 25]. One of the strong motivations to study the $\gamma^{*} B \rightarrow B^{\prime}$ reactions is to clarify the role of the meson cloud dressing, which is of fundamental importance, as was demonstrated by the $\gamma^{*} N \rightarrow \Delta$ reaction [1, 26 30]. The data, except for the $\gamma^{*} N \rightarrow \Delta$ reaction, namely the $\Sigma^{* 0} \rightarrow \gamma \Lambda$ and $\Sigma^{*+} \rightarrow \gamma \Sigma^{+}$decay widths, have become available only recently 31 34]. In general, most of the model predictions significantly underestimate the data, particularly those for the $\Sigma^{*+} \rightarrow \gamma \Sigma^{+}$decay width (see Ref. [35] for a more detailed discussion).

In our previous work [35] we studied the $\gamma^{*} B \rightarrow B^{\prime}$ re- actions using a covariant constituent quark model, complemented by the pion cloud effects extrapolated by the $\gamma^{*} N \rightarrow \Delta$ reaction based on an $\mathrm{SU}(3)$ symmetry. The pion cloud effects were included in the leading order, namely, they included only the processes with the direct photon coupling to the pion. The electromagnetic transition form factors calculated were decomposed into the valence quark and pion cloud contributions. We concluded that the pion cloud effects could help to explain satisfactorily the $\gamma^{*} N \rightarrow \Delta$ data, but only partially help to explain the data for the $\gamma^{*} \Lambda \rightarrow \Sigma^{* 0}$ and $\gamma^{*} \Sigma^{+} \rightarrow \Sigma^{*+}$ reactions. Therefore, the other effects, such as the contributions from the heavier mesons like the kaon, and alternative higher order processes involving the meson cloud, may be relevant to explain the experimental decay widths. The next-order processes to be included in this study are the processes which one photon couples to the intermediate baryon states while one meson is in the air. In addition, the heavier mesons to be taken into account are the kaon and eta meson, the next lighter mesons to the pion.

In a model with pointlike quarks the photon coupling with the intermediate baryon states is not expected to be important for the meson cloud contributions, since the octet to the decuplet electromagnetic transitions are dominated by the magnetic interactions, and the quark anomalous magnetic moments vanish for the pointlike quarks. However, in a constituent quark model like the one we use in this study, the covariant spectator quark model, the octet-decuplet electromagnetic transitions are dominated by the mechanisms with a quark spin flip (magnetic type interactions). Therefore, the valence quark contributions may be very important when the quark anomalous magnetic moments are significant. Furthermore, we can expect important meson cloud contributions from the intermediate octet-decuplet baryon electromagnetic transitions while one meson is in the air (photon-vertex correction), since these mechanisms also depend on the quark anomalous magnetic moments. 


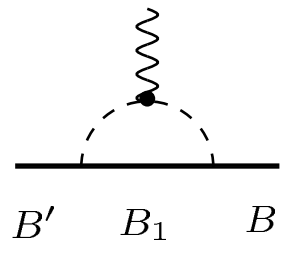

(a) (b)

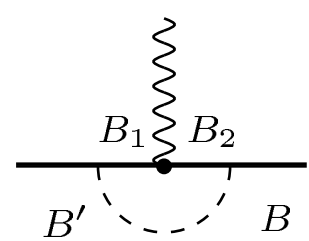

FIG. 1: Meson cloud contributions for the electromagnetic transition form factors. Between the initial octet $(B)$ and final decuplet $\left(B^{\prime}\right)$ baryon states, there are several possible intermediate baryon states: (a) $B_{1}$; (b) $B_{1}$ and $B_{2}$. Depending on the meson $M$, mesonbaryon states $\left(M-B_{1}\right.$ and $\left.M-B_{2}\right)$ may arise, where $B_{1}$ and $B_{2}$ are the octet and decuplet baryon states in this study.

In this work we improve the calculation of the meson cloud contributions for the $\gamma^{*} B \rightarrow B^{\prime}$ transitions made in the previous work [35], and predict the corresponding electromagnetic decay widths (determined at $Q^{2}=0$ ). The improvements are the following: i) inclusion of the photon coupling to the intermediate baryon states; ii) inclusion of the effects of the heavier meson clouds, kaon and eta meson, besides the pion. As in the previous work, the transition form factors can be decomposed into the valence quark and meson cloud contributions. The processes included as the meson cloud contributions in this work are depicted in Fig. 1, in terms of the meson and baryon degrees of freedom.

We will conclude that the effects of the intermediate baryon states combined with the kaon cloud, improve the agreement of our model with the experimental data.

The valence quark contributions are estimated based on the covariant spectator quark model [36 40] as in the previous work. Thus, the baryons are described as threequark systems. The valence quark contributions for the transition form factors are calculated using the octet and decuplet baryon wave functions and the quark electromagnetic current of the model, determined in the previous works.

To describe the meson cloud contributions for the octet-decuplet baryon electromagnetic transitions we need a microscopic model to describe the virtual mesonbaryon states.

Contrary to the valence quark contributions that dominate in the large $Q^{2}$ region, the meson cloud effects are long-range processes, and are known to be of crucial to explain the transition helicity amplitudes and form factors in the low $Q^{2}$ region [1, 2]. To incorporate the meson cloud effects, we use the cloudy bag model (CBM) [15, 41 44] which treats the mesons as pointlike particles to describe the meson cloud dressing in the static approximation for the baryons. All such approximations have been practiced well in the past within the CBM, and may be regarded as under control.

Although the CBM framework differs from the covari- ant spectator quark model for the treatment of the valence quarks, the CBM can be used as an effective description of the long-range physics of meson cloud dressing. The possible conflict between the two models e.g., the lack of the explicit covariance and the limitation of the applicability for the large $Q^{2}$ region in the CBM, can be overcome in a proper manner, since one can define a covariant extension of the model based on covariant parametrizations for the meson cloud contributions that are equivalent with the CBM result at $Q^{2}=0$. See for instance Refs. [45 47], where meson cloud contributions were estimated in different reactions. In addition, the merit of using the CBM is that the model is based on $\mathrm{SU}(3)$ (SU(6) flavor-spin) symmetry and chiral symmetry.

The explicit calculation of the meson cloud contributions considered in this study requires two kind of mechanisms. The first mechanism is the photon coupling with the meson, and for this, we use a formalism similar to that applied in Ref. [35]. However, in the present study we take into account the explicit dependence of the baryon and meson (pion, kaon and eta) masses. [Previously, we used SU(3) symmetry for the baryon masses, and only the pion cloud was included.] The second mechanism is the photon coupling with the intermediate baryon states, which is more delicate and model dependent, since this requires an estimate of all the intermediate octet-octet, octet-decuplet decuplet-octet and decuplet-decuplet transition form factors at $Q^{2}=0$. The corresponding expressions are derived in the CBM framework, but since we describe the valence quark cores with the covariant spectator quark model, it is necessary to reinterpret the CBM quark magnetic moments in terms of those calculated by the spectator quark model. This will be done using $\mathrm{SU}(3)$ symmetry to be explained in detail later.

Finally, the results from the CBM are normalized by the pion cloud contribution obtained in the covariant spectator quark model for the $\gamma^{*} N \rightarrow \Delta$ transition [27], under the assumption that the pion cloud is the dominant meson cloud contribution. With this procedure also used in the previous work [35], we preserve the parametrization of the covariant spectator quark model for the core, and estimate the effects of the meson cloud for the other octet to decuplet transitions, as well as the kaon and eta clouds for the $\gamma^{*} N \rightarrow \Delta$ reaction.

This article is organized as follows: in Sec. II we explain the decomposition of the valence and meson cloud contributions for the transition form factors. In Sec. III we review the formalism associated with the valence quark contributions for the form factors, and express the results in terms of effective quark magnetic moments, which are also necessary for the calculation of the meson cloud effects. In Sec. IV we present the formalism associated with the meson cloud dressing. The results are presented in Sec. $\mathrm{V}$, while the final conclusions are given in Sec. VI, 


\section{FORMALISM}

Next, we discuss briefly the formalism necessary to describe the valence quark contributions, as well as the mechanism of the meson cloud dressing.

In the covariant spectator quark model, baryons are treated as three-quark systems [36 40]. The electromagnetic interactions with the baryons are described by the photon coupling with the constituent quarks in the relativistic impulse approximation, and the quark electromagnetic structure is represented in terms of the quark form factors parameterized by a vector meson dominance mechanism [37, 40]. The parametrization of the quark current, calibrated previously in the studies of the nucleon form factors [37] and by the lattice QCD data for the decuplet baryons [40], encodes effectively the gluon and quark-antiquark substructure of the constituent quarks. The baryon wave functions derived from the $\mathrm{SU}(6) \otimes \mathrm{O}(3)$ structure, are written in terms of an offshell quark, that is free to interact with the photon fields, and two on-shell quarks. Integrating over the quark-pair degrees of freedom, we reduce the three-quark baryon state to a quark-diquark state, where the diquark can be represented as an on-shell spectator particle with an effective mass of $m_{D}$ [37, 38, 40].

Under the assumption that each baryon system can be described by the wave function with an S-state configuration for the quark-diquark system in the first approximation, we calculated the valence quark contributions for the magnetic form factors $G_{M}^{B}$ in the previous work [35], where the upper index $B$ labels the contributions from the quark core (bare), using the wave functions from Refs. 40, 45]. (See Ref. [35] for more details.) Contributions from the electric and Coulomb quadrupole form factors appear only beyond the S-state approximation for the decuplet baryon wave functions. However, their contributions are expected to be small (small orbital angular momentum admixtures) [28, 30], and thus they are neglected in this work.

As mentioned already, the constituent quarks considered in this work, have internal structure, and the structure is encoded in a vector meson dominance parametrization [37, 39, 40], that includes effectively, among other effects, the meson cloud dressing of the quarks. However, it should be emphasized that there are meson cloud effects that cannot be included in the constituent quark structure, such as the process of meson exchange between the different quarks inside the baryon, which cannot be reduced to a simple diagram of a quark dressing. Processes of this kind, have to be represented at the hadronic level (meson and baryon states) as the diagrams shown in Fig. 1. Thus, the meson cloud in this study, is regarded as a process of one meson exchange between the different quarks inside the baryon [39]. Since the meson cloud dressing can appear in two independent mechanisms (self dressing of the quarks and the others) there is no double counting. In summary, besides the contributions from the valence quark core calculated using the quark electromagnetic form factors, there are meson cloud effects that have to be taken into account in the electromagnetic transitions between the baryon states. Those meson cloud effects are the main focus of the present work.

From the discussions made previously, we conclude that the magnetic transition form factors $\left(G_{M}\right)$ can be represented as the sum of the valence quark $\left(G_{M}^{B}\right)$ and meson cloud $\left(G_{M}^{\mathrm{MC}}\right)$ contributions in the present approach: $G_{M}=G_{M}^{B}+G_{M}^{\mathrm{MC}}$. In particular, for the study of the baryon decuplet decay widths, we need to consider only the case $Q^{2}=0$. Thus, we can write

$$
G_{M}(0)=G_{M}^{B}(0)+G_{M}^{\mathrm{MC}}(0) .
$$

As mentioned already, the meson cloud contribution for the octet to decuplet transition, can be decomposed in the two processes displayed in Fig. 11 for the first and second order, classified by the number of the baryon propagators. The diagram (a) represents the direct coupling of a photon with the intermediate state meson (first order, one baryon propagator). The diagram (b) represents the direct coupling of a photon with the intermediate state baryons (second order, two baryon propagators). One can then decompose $G_{M}^{\mathrm{MC}}(0)$ into the contributions from the diagrams (a) and (b):

$$
G_{M}^{\mathrm{MC}}(0)=G_{M}^{\mathrm{MCa}}(0)+G_{M}^{\mathrm{MCb}}(0) .
$$

The meson cloud contributions corresponding to each diagram (a) and (b), can be further decomposed into the pion, kaon, and eta cloud contributions.

As in the previous work [35], in order to keep the parametrization of the covariant spectator quark model, we regularize the results for the pion cloud contribution by that from the covariant spectator quark model for the $\gamma^{*} N \rightarrow \Delta$ reaction,

$$
G_{M}^{\mathrm{MC} \pi}(0)=3 \lambda_{\pi},
$$

where $\lambda_{\pi}=0.441$ defines the strength of the pion cloud effect 28]. In this procedure we assume that the pion cloud is the dominant meson cloud effect in the $\gamma^{*} N \rightarrow \Delta$ reaction. Later we will see that this assumption is indeed justified. For the other octet to decuplet transition reactions, and also for all the meson clouds considered in the present work, we use the relation,

$$
G_{M}^{\mathrm{MC}}(0)=f_{B B^{\prime}}\left(3 \lambda_{\pi}\right),
$$

where the factor $f_{B B^{\prime}}$ contains the pion, kaon and eta meson cloud contributions from the both diagrams (a) and (b) in Fig. 1. The calculation of the coefficient $f_{B B^{\prime}}$ will be explained in Sec. V

The diagram (b) includes in the intermediate states, the octet-octet, octet-decuplet, decuplet-octet and decuplet-decuplet baryon electromagnetic transitions. Therefore, to estimate the contributions from the possible intermediate baryon state transitions, we need to calculate all the corresponding transition magnetic form 
factors at $Q^{2}=0$. One can in principle calculate them in the covariant spectator quark models for this purpose, however, the explicit estimates corresponding to all the intermediate baryon state transitions would be complex and tedious. Therefore, for the diagram (b) we use the estimate made in the $\mathrm{CBM} / \mathrm{SU}(6)$ framework, where all the intermediate state contributions can be related with the valence quark magnetic moments. In order to relate the $\mathrm{CBM} / \mathrm{SU}(6)$ quark magnetic moments with the anomalous magnetic moments in the covariant spectator quark model, we will start by reviewing the expressions used for the valence quark contributions for the octet to decuplet electromagnetic transition form factors.

\section{VALENCE QUARK CONTRIBUTIONS}

In the covariant spectator quark model the valence quark contributions for the form factors are calculated using the octet and decuplet baryon wave functions, and the constituent quark current. The quark current has the general form [37, 40],

$$
j_{q}^{\mu}\left(Q^{2}\right)=j_{1}\left(Q^{2}\right) \gamma^{\mu}+j_{2}\left(Q^{2}\right) \frac{i \sigma^{\mu \nu} q_{\nu}}{2 M_{N}},
$$

where $j_{i}(i=1,2)$ are the quark form factors that can be parametrized in terms of a vector dominance mechanism. The form factors $j_{i}$ can also be decomposed in the quark-isoscalar, quark-isovector and strange-quark components. The details can be found in Refs. [35, 37, 39, 40], but are not important for the present discussion, since we are considering the $Q^{2}=0$ case, where $j_{1}(0)=e_{q}$ and $j_{2}(0)=e_{q} \kappa_{q}$. The last equation defines the quark anomalous moment $\left(\kappa_{q}\right)$ in the covariant spectator quark model formalism.

To calculate the transition form factors, we project the operator $j_{i}$ on the mixed anti-symmetric $\left(\left|M_{A}\right\rangle\right)$ and mixed symmetric $\left(\left|M_{S}\right\rangle\right)$ components of the octet and (fully symmetric) decuplet $\left|B^{\prime}\right\rangle$ flavor states:

$$
\begin{aligned}
j_{i}^{A} & =3\left\langle B^{\prime}\left|j_{i}\right| M_{A}\right\rangle, \\
j_{i}^{S} & =3\left\langle B^{\prime}\left|j_{i}\right| M_{S}\right\rangle .
\end{aligned}
$$

More details can be found in Refs. 35, 39, 40]. Note that, for the octet to decuplet baryon transitions only the components $j_{i}^{S}$ (isovector) are relevant. Finally, the magnetic form factor can be written [35] as,

$$
G_{M}^{B}=\frac{2 \sqrt{2}}{3} \sqrt{\frac{2}{3}} \bar{f}_{v} \mathcal{I}
$$

with

$$
\bar{f}_{v}=\frac{2 M_{B}}{M_{B^{\prime}}+M_{B}}\left\{\frac{j_{1}^{S}}{\sqrt{2}}+\frac{M_{B^{\prime}}+M_{B}}{2 M_{N}} \frac{j_{2}^{S}}{\sqrt{2}}\right\},
$$

where the coefficients $\frac{1}{\sqrt{2}} j_{i}^{S}$ can be found in Ref. [35], and $\mathcal{I}$ is the overlap integral between the octet $\left(\psi_{B}\right)$ and decuplet $\left(\psi_{B^{\prime}}\right)$ radial wave functions (see details also in Ref. [35]). The radial wave functions $\psi_{B}$ and $\psi_{B^{\prime}}$ are scalar functions of the baryon and diquark momenta 35 , 39, 40, 45]. In Eqs. (3.4) and (3.5) $G_{M}^{B}, \bar{f}_{v}$ and $\mathcal{I}$ are exclusive functions of $Q^{2}$.

Now, we focus again on the $Q^{2}=0$ case. In this case we can write the factor $\bar{f}_{v}$ in a more compact form, defining the effective quark magnetic moment of the transition $\gamma^{*} B \rightarrow B^{\prime}$ as

$$
\hat{\mu}_{q}=\frac{2 M_{B}}{M_{B^{\prime}}+M_{B}}+\frac{M_{B}}{M_{N}} \kappa_{q} .
$$

Note that the expression for $\hat{\mu}_{q}$ is reduced to the usual form, $\mu_{q}=\left(1+\kappa_{q}\right)$, in the limit $M_{B^{\prime}}=M_{B}=M_{N}$. However, $\hat{\mu}_{q}$ now depends on the masses of the "submultiplets" $\left(M_{B^{\prime}}\right.$ and $M_{B}$ in the $\gamma^{*} B \rightarrow B^{\prime}$ transition). We keep this dependence in mind, but suppress the indices $B$ and $B^{\prime}$ in $\hat{\mu}_{q}$ for simplicity.

The explicit expressions for $\bar{f}_{v}$ in terms of $\hat{\mu}_{q}$ are presented in Table I. In particular, we can express the reactions involving the $\Sigma$ and $\Xi$ as,

$$
\bar{f}_{v}=\frac{1}{6}\left(2 \hat{\mu}_{u}-\hat{\mu}_{d}+2 \hat{\mu}_{s}\right)+\frac{1}{6}\left(2 \hat{\mu}_{u}+\hat{\mu}_{d}\right) t_{3},
$$

where $t_{3}=J_{3}$ for $\Sigma$ and $t_{3}=\tau_{3}$ for $\Xi$. The matrices $J_{3}=\operatorname{diag}(1,0,-1)$ and $\tau_{3}=\operatorname{diag}(1,-1)$, are, respectively, isospin- 1 and isospin- $1 / 2$ operators that act on the isospin states of the baryons $B$ and $B^{\prime}$.

The contributions from the valence quarks for the form factors can also be estimated by the SU(6) quark model in terms of the quark magnetic moments $\mu_{q}$. The results are expressed in terms of the $u, d$ and $s$ quark magnetic moments, $\mu_{u}, \mu_{d}$ and $\mu_{s}$, respectively. The CBM uses the spin-flavor $\mathrm{SU}(6)$ wave functions, but calculates the values of $\mu_{q}$ using the CBM (MIT bag) formalism. Since usually $\mu_{u} \equiv \mu_{d}$ in the CBM (reflecting the quark masses used, $m_{u}=m_{d}$ ), we use $\bar{\mu}_{u}$ to represent either $\mu_{u}$ or $\mu_{d}$.

Note that the definitions of the quark magnetic moments discussed here do not include the quark charges, contrarily to the convention used for instance in naive quark models [48, 49].

The results for $G_{M}^{B}$ (quark core contributions) from the CBM are presented in the last column in Table I. For the $\gamma^{*} N \rightarrow \Delta$ reaction the result is [8, 26]

$$
G_{M}^{B}(0)=\frac{2 \sqrt{2}}{3} \bar{\mu}_{u}, \quad(\mathrm{SU}(6)),
$$

where $\bar{\mu}_{u}=\mu_{p}$, the proton magnetic moment in the $\mathrm{SU}(2)$ limit $^{1}$. In this limit also $\mu_{n}=-\frac{2}{3} \bar{\mu}_{u}$.

\footnotetext{
${ }^{1}$ For simplicity we ignore the factor $\sqrt{\frac{M_{N}}{M_{\Delta}}}$ that transforms magnetic moment $\mu_{N \Delta}$ into the corresponding form factor $G_{M}(0)$. This simplification has no consequence in the present work, since to identify the results of the CBM and those of the spectator quark model, global factors are not important.
} 


\begin{tabular}{|c|c|c|c|}
\hline & $\bar{f}_{v}$ & $G_{M}^{B}$ & $G_{M}^{B}(\mathrm{CBM})$ \\
\hline$\gamma^{*} p \rightarrow \Delta^{+}$ & $\frac{1}{3}\left(2 \hat{\mu}_{u}+\hat{\mu}_{d}\right)$ & $A \frac{1}{3}\left(2 \mu_{u}+\mu_{d}\right)$ & $A \bar{\mu}_{u}$ \\
\hline$\gamma^{*} n \rightarrow \Delta^{0}$ & $\frac{1}{3}\left(2 \hat{\mu}_{u}+\right.$ & $A \frac{1}{3}\left(2 \mu_{u}+\mu_{d}\right)$ & $A \bar{\mu}_{u}$ \\
\hline$\gamma^{*} \Lambda \rightarrow \Sigma^{* 0}$ & $\sqrt{\frac{3}{4}} \frac{1}{3}\left(2 \hat{\mu}_{u}+\hat{\mu}_{d}\right)$ & $\sqrt{\frac{3}{4}} A \frac{1}{3}\left(2 \mu_{u}+\mu_{d}\right)$ & $\sqrt{\frac{3}{4}} A \bar{\mu}_{u}$ \\
\hline$\gamma^{*} \Sigma^{+} \rightarrow \Sigma^{*+}$ & $\frac{1}{3}\left(2 \hat{\mu}_{u}+\hat{\mu}_{s}\right)$ & $A \frac{1}{3}\left(2 \mu_{u}+\mu_{s}\right)$ & $A \frac{1}{3}\left(2 \bar{\mu}_{u}+\mu_{s}\right)$ \\
\hline$\gamma^{*} \Sigma^{0} \rightarrow \Sigma^{* 0}$ & $\frac{1}{6}\left(2 \hat{\mu}_{u}-\hat{\mu}_{d}+2 \hat{\mu}_{s}\right)$ & $A \frac{1}{6}\left(2 \mu_{u}-\mu_{d}+2 \mu_{s}\right)$ & $A \frac{1}{3}\left(\bar{\mu}_{u}+2 \mu_{s}\right)$ \\
\hline$\gamma^{*} \Sigma^{-} \rightarrow \Sigma^{*-}$ & $\frac{1}{3}\left(-\hat{\mu}_{d}+\hat{\mu}_{s}\right)$ & $A \frac{1}{3}\left(-\mu_{d}+\mu_{s}\right)$ & $A \frac{1}{3}\left(-\bar{\mu}_{u}+\mu_{s}\right)$ \\
\hline$\gamma^{*} \Xi^{0} \rightarrow \Xi^{* 0}$ & $\frac{1}{3}\left(2 \hat{\mu}_{u}+\hat{\mu}_{s}\right)$ & $A \frac{1}{3}\left(2 \mu_{u}+\mu_{s}\right)$ & $A \frac{1}{3}\left(2 \bar{\mu}_{u}+\mu_{s}\right)$ \\
\hline$\gamma^{*} \Xi^{-} \rightarrow \Xi^{*-}$ & $\frac{1}{3}\left(-\hat{\mu}_{d}+\hat{\mu}_{s}\right)$ & $A \frac{1}{3}\left(-\mu_{d}+\mu_{s}\right)$ & $A \frac{1}{3}\left(-\bar{\mu}_{u}+\mu_{s}\right)$ \\
\hline
\end{tabular}

TABLE I: Coefficients $j_{i}^{S}(i=1,2), \bar{f}_{v}$ and valence quark contributions for the $G_{M}$ form factors. In the expressions for $G_{M}^{B}$, one has $A=\frac{2 \sqrt{2}}{3}$.

In order to compare the results of the covariant spectator quark model with those of the CBM, we need to relate the spectator model quark magnetic moments with those of the CBM/SU(6). Motivated by Eq. (3.8), and taking into account that the structure of $G_{M}^{B}$ in the covariant spectator quark model, given by Eqs. (3.4) and (3.5), we define $\mu_{q} \equiv \sqrt{\frac{2}{3}} \hat{\mu}_{q} \mathcal{I}(0)$, or

$$
\mu_{q}=\sqrt{\frac{2}{3}}\left\{\frac{2 M_{B}}{M_{B^{\prime}}+M_{B}}+\frac{M_{B}}{M_{N}} \kappa_{q}\right\} \mathcal{I}(0),
$$

for the covariant spectator quark model.

With the above identification of the quark magnetic moments, we can write the $\gamma^{*} N \rightarrow \Delta$ magnetic form factor in the covariant spectator quark model as

$$
G_{M}^{B}(0)=\frac{2 \sqrt{2}}{3} \frac{1}{3}\left(2 \mu_{u}+\mu_{d}\right), \quad \text { (Spectator). }
$$

Note the similarity between Eqs. (3.8) and (3.10). If we replace $\frac{1}{3}\left(2 \mu_{u}+\mu_{d}\right) \rightarrow \bar{\mu}_{u}$ as in the $\mathrm{SU}(2)$ symmetric case, the two equations are equivalent. The expressions for the other octet to decuplet transitions are presented in Table 【, with $A=\frac{2 \sqrt{2}}{3}$. Also for the other reactions the expressions from the covariant spectator quark model and CBM are equivalent in the $\mathrm{SU}(2)$ symmetric limit, although in the case of the covariant spectator quark model $\mu_{q}$ varies from reaction to reaction.

Thus, we can relate the $\mathrm{CBM} / \mathrm{SU}(6)$ results of the bare core (valence quark contributions) with those of the covariant spectator quark model defining the quark magnetic moments by Eq. (3.9). The quark magnetic moments of the covariant spectator quark model generalize the usual magnetic moments by the inclusion of the octet and decuplet baryon mass dependence $\left(M_{B}\right.$ and $\left.M_{B^{\prime}}\right)$. Therefore, the effective quark magnetic moment $\mu_{q}$, defined by Eq. (3.9), differs from transition to transition in the covariant spectator quark model. However, as mentioned already, the familiar expression is recovered in the limit $M_{B^{\prime}}=M_{B}=M_{N}$, apart from some constants.
Another interesting point is the dependence of $\mu_{q}$ on the overlap integral $\mathcal{I}(0)$, which is a consequence of the difference between the octet and decuplet radial wave functions of the transition. In a naive picture with $M_{B}=$ $M_{B^{\prime}}$, the octet and decuplet radial wave functions can be approximated by the same radial wave function (defined in the same frame) and the overlap integral would be $\mathcal{I}(0)=1$. In the present case as discussed in Ref. [35], $\mathcal{I}(0)$ is about $0.8-0.9$, depending on the transitions.

A note is in order about the $\mathrm{SU}(6)$ result for $G_{M}^{B}(0)$, given by Eq. (3.8) for the $\gamma^{*} N \rightarrow \Delta$ reaction. The numerical result using the experimental value for $\mu_{p}$, is $G_{M}^{B}(0)=2.3$ (including the effect of the nucleon and $\Delta$ masses, see footnote 1). This result, overestimates the relativistic calculations. Some relativistic calculations take into account the differences between the nucleon and $\Delta$ masses and also the nonzero momentum of the nucleon at $Q^{2}=0$ in the $\Delta$ rest frame. The Sato-Lee model [50] for instance gives $G_{M}^{B}(0)=2.05$. As for the covariant spectator quark model, we recall that the model predicts the upper limit of $G_{M}^{B}(0)=2.07$ [27, 35], but in practice this value is reduced by the overlap of the nucleon and $\Delta$ radial wave functions, $\mathcal{I}(0)$, which is always smaller than unity as already mentioned. See Appendix B in Ref. [35] for details. For the present study it is not important even if our expressions differ from those of the $\mathrm{SU}(6)$ by a factor. For example, the factor $\sqrt{\frac{2}{3}}$ may be a consequence of the relativistic calculation. Also the overlap integral $\mathcal{I}(0)$, does not appear in the simple $\mathrm{SU}(6)$ quark model expressions, due to the static approximation $[\mathcal{I}(0) \rightarrow 1]$. The important point is to establish the correspondence between the analytical expressions in the $\mathrm{SU}(6)$ quark model and those of the spectator formalism consistently.

Next, we comment on the renormalization of the baryon wave functions. In the present calculation we use the decuplet baryon radial wave functions from Ref. [40] and those of the octet baryons from Ref. [45]. In these cases the decuplet baryon wave functions were determined assuming that they have no meson cloud dressing, 
while the octet baryon wave functions were determined assuming a small pion cloud dressing. As discussed already in Ref. [35], the correction due to the renormalization of the octet baryon wave functions (due to the pion cloud dressing) is small, and can be neglected in a first approximation (less than $4 \%$ effect). In the present work we include kaon and a eta clouds in addition to the pion cloud. Although we cannot calculate the renormalization effects due to these mesons for the baryon wave functions in the covariant spectator quark model framework, we will assume, as was already done for the pion could, that the meson cloud effects are small and can be neglected in the normalization of the wave functions in a first approximation. Later we will discuss the renormalization effect due to the meson cloud effects, since the meson cloud contributions depend also on the wave functions.

\section{MESON CLOUD CONTRIBUTIONS}

To estimate the meson cloud contributions for the processes shown by the diagrams (a) and (b) in Fig. 1. we apply the CBM [41]. As usually practiced in the CBM, we use the static approximation and neglect the momentum of the baryons in the initial, intermediate and final baryon states, by replacing the respective energies by their masses 15, 41 43. The same approximation is also used in the heavy baryon chiral perturbation theory [22, 51 53]. In addition, we ignore the possible center-of-mass correction for the 3-quark composite baryon (core) systems, keeping in mind that this correction reduces the bare core transition amplitudes for the $\gamma^{*} N \rightarrow \Delta$ reaction by 5 to $10 \%$ in the region $Q^{2} \lesssim 0.5$ $\mathrm{GeV}^{2}$ [15]. The effects are expected to be even smaller for the remaining reactions, since the corresponding baryons are heavier.

Although the approximations discussed above break the Lorentz covariance, the phenomenological successes and practices in describing the physics in the low $Q^{2}$ region [15, 41 43], suggest that the approximations may be well under control in the present study, particularly at $Q^{2}=0$ (small kinematic corrections).

To carry out the calculations of the meson cloud contributions, all the intermediate states are summed over utilizing the standard angular momentum algebra in flavor and spin spaces combined with the Wigner-Eckart theorem [41 43]. Thus, the summation is made based on the SU(6) symmetry at the flavor-spin wave function level. The SU(3) breaking effects are, partially included using the physical baryon and meson masses, and via the quark masses, $m_{u}=m_{d} \neq m_{s}$. Note that in the covariant spectator quark model the $\mathrm{SU}(3)$ symmetry is explicitly broken in the octet and decuplet baryon wave functions.

The equations derived in the CBM for $Q^{2}=0$, depend only on one-dimensional integrals. In some cases, the CBM integrals have singularities in the integrand func- tions (poles associated with physical baryons or mesons in the intermediate states). These poles yield imaginary parts for the calculated integrals. For simplicity we evaluate those integrals using the principal value integral. Based on the results from the CBM [15] for the $\gamma^{*} N \rightarrow \Delta$ reaction, we may expect the imaginary part to be about $15-20 \%$ of the real part near $Q^{2}=0$. Since the decay width depends on $\left|G_{M}(0)\right|^{2}$, this approximation has only a small effect in the final results (a 20\% imaginary part of the real part on $G_{M}(0)$ leads to a $4 \%$ correction for $\left.\left|G_{M}(0)\right|^{2}\right)$.

\section{A. Direct coupling with the meson}

We first consider the contributions from the processes represented by the diagram (a) in Fig. 1. In the following the upper index $M$ stands for the meson $(M=\pi, K)$. The CBM loop integral functions [41, 42] for the initial $(B)$ and final $\left(B^{\prime}\right)$ baryons that depend also on the intermediate baryon $B_{1}$ states, for the pion and kaon cloud diagrams, will be denoted by $H_{B B^{\prime}}^{\pi}\left(B_{1}\right)$ and $H_{B B^{\prime}}^{K}\left(B_{1}\right)$, respectively.

The contributions from the diagram (a) for the $\gamma^{*} B \rightarrow$ $B^{\prime}$ can be written as

$$
G_{M}^{\mathrm{MCa} a}=\sum_{M, B_{1}} C_{B B^{\prime} ; B_{1}}^{M} H_{B B^{\prime}}^{M}\left(B_{1}\right),
$$

where $C_{B B^{\prime} ; B_{1}}^{M}$ are the coefficients calculated in the CBM framework, and are presented in Appendix $\mathrm{A}$

The explicit expression for $H_{B B^{\prime}}^{M}\left(B_{1}\right)$ is,

$$
\begin{aligned}
& H_{B B^{\prime}}^{M}\left(B_{1}\right)=\frac{1}{12 \pi^{2}}\left(\frac{f_{\pi N N}}{m_{\pi}}\right)^{2} \\
& \times \int_{0}^{\infty} d k\left\{\frac{k^{4}\left[j_{0}(k R)+j_{2}(k R)\right]^{2}}{\omega_{k}\left[4 \omega_{k}^{2}-\left(M_{B^{\prime}}-M_{B}\right)^{2}\right]}\right. \\
& \left.\times \frac{4 \omega_{k}+2 M_{B_{1}}-M_{B^{\prime}}-M_{B}}{\left(M_{B_{1}}-M_{B}+\omega_{k}\right)\left(M_{B_{1}}-M_{B^{\prime}}+\omega_{k}\right)}\right\},
\end{aligned}
$$

where $R$ is the bag radius, $j_{l}(l=0,2)$ are the spherical Bessel functions arising from the CBM form factor, $\omega_{k}=\sqrt{m_{M}^{2}+k^{2}}$ for $M=\pi, K$ is the meson energy, and $f_{\pi N N}$ is the pion-nucleon coupling constant. As already mentioned the integral symbol should be read as the principal value integral.

In the present work we take a typical, successful value for the bag radius, $R=1 \mathrm{fm}$ [43]. The dependence of the calculated quantities on the values of the bag radius chosen, can be found in Refs. [15, 41, 42].

The factor $\left(\frac{f_{\pi_{N N}}}{m_{\pi}}\right)^{2}$ is included in the loop integral definition for all baryon and meson cases, since all the couplings are redefined in terms of $f_{\pi N N}$. For discussions about the renormalized $f_{\pi N N}$ value used in the CBM, see Ref. [15, 42]. 


\begin{tabular}{lccccc}
\hline \hline & $G_{M}^{B}(0)$ & $G_{M}^{\mathrm{MCa} \pi}(0)$ & $G_{M}^{\mathrm{MCb} \pi}(0)$ & $G_{M}^{\mathrm{MC} \pi}(0)$ & $G_{M}(0)$ \\
\hline \hline CBM $\left[\tilde{G}_{M}(0)\right]$ & 1.633 & 0.883 & 0.754 & 1.634 & 3.270 \\
Spectator $\left[G_{M}(0)\right]$ & 1.633 & 0.713 & 0.610 & 1.323 & 2.956 \\
\hline \hline
\end{tabular}

TABLE II: Pion cloud contributions for the $\gamma^{*} N \rightarrow \Delta$ reaction. The quantities with the superscript $\pi$ refer only to the pion cloud. The first entry includes the results from the CBM, while the second includes the corresponding quantities from the covariant spectator quark model.

\section{B. Coupling with intermediate baryon states}

Next, we consider the contributions from the diagram (b), due to the clouds of the pion, kaon and $\eta$ meson. Since the processes depend on the intermediate baryon states $B_{1}$ and $B_{2}$, the respective contributions generally depend on the intermediate state transition form factors between $B_{1}$ and $B_{2}$, and these can, in the $\mathrm{SU}(6)$ quark model, be represented by the combinations of the quark magnetic moments $\mu_{q}$.

In order to obtain a simple estimate for the meson cloud contributions without explicitly summing over a huge number of the intermediate states, we use a technique developed and used in the CBM framework [43] with the exact isospin symmetry, $\mu_{u}=\mu_{d}$. The use of the isospin symmetry simplifies the calculation drastically by reducing the number of terms to be considered, and can be justified when the difference between $\mu_{u}$ and $\mu_{d}$ is small.

In the following calculations of the meson cloud effects we will replace the CBM quark magnetic moments by these of the covariant spectator quark model as defined by Eq. (3.9). In order to keep the isospin symmetry in those calculations we replace $\mu_{u}$ and $\mu_{d}$ by an average $\bar{\mu}_{u}$ to be defined later.

Then, the contributions from the diagram (b) for the $\gamma^{*} B \rightarrow B^{\prime}$ transition can be written as

$$
G_{M}^{\mathrm{MBb}}=\sum_{M, B_{1}, B_{2}} D_{B B^{\prime} ; B_{1} B_{2}}^{M} H_{B B^{\prime}}^{2 M}\left(B_{1}, B_{2}\right),
$$

where the CBM-based integral is represented by $H_{B B^{\prime}}^{2 M}$ to be defined next, and $D_{B B^{\prime} ; B_{1} B_{2}}^{M}$ are the coefficients which depend on the effective magnetic moments $\mu_{q}$. The expressions for $D_{B B^{\prime} ; B_{1} B_{2}}^{M}$ are given in Appendix $\mathrm{A}$.

The integral $H_{B B^{\prime}}^{2 M}$ is defined by

$$
\begin{aligned}
& H_{B B^{\prime}}^{2 M}\left(B_{1}, B_{2}\right)=\frac{1}{12 \pi^{2}}\left(\frac{f_{\pi N N}}{m_{\pi}}\right)^{2} \\
& \times \int_{0}^{\infty} d k\left\{\frac{k^{4}\left[j_{0}(k R)+j_{2}(k R)\right]^{2}}{\omega_{k}\left(M_{B_{1}}-M_{B}+\omega_{k}\right)\left(M_{B_{2}}-M_{B^{\prime}}+\omega_{k}\right)}\right\} .
\end{aligned}
$$

Again, the principal value integration should be understood. In Eq. (4.4) $B_{1}$ and $B_{2}$ are the baryons in the intermediate states with masses $M_{B_{1}}$ and $M_{B_{2}}$, respectively, and the upper index $2 M$ indicates that there are two baryon propagators while a meson $M$ is in the air.
Besides that, the functions are obtained with a static approximation, the same as for the $H_{B B^{\prime}}^{M}\left(B_{1}\right)$ case, but we also have now $\omega_{k}=\sqrt{m_{\eta}^{2}+k^{2}}$ when $M=\eta$.

To be consistent with the $\mathrm{SU}(2)$ symmetry in the calculation of the function $D_{B B^{\prime} ; B_{1} B_{2}}^{M}$ for the meson cloud contributions, we replace in the expressions for the magnetic moments, $\mu_{u}$ and $=\mu_{d}$, by the average,

$$
\bar{\mu}_{u} \equiv \frac{1}{3}\left(2 \mu_{u}+\mu_{d}\right) .
$$

With this definition, the results for the core contributions are the same for the CBM and the covariant spectator quark model for the reactions $\gamma^{*} N \rightarrow \Delta$ and $\gamma^{*} \Lambda \rightarrow \Sigma^{* 0}$. The same expression will be used for the calculation of the meson cloud contributions represented by the dia$\operatorname{gram}(\mathrm{b})$.

As for the reactions involving the $\Sigma$ and $\Xi$ in the calculation of the meson cloud effects, we use the replacement suggested by Eq. (3.7),

$$
\begin{aligned}
& \frac{1}{6}\left(2 \mu_{u}-\mu_{d}+2 \mu_{s}\right)+\frac{1}{6}\left(2 \mu_{u}+\mu_{d}\right) t_{3} \\
& \rightarrow \frac{1}{6}\left(\bar{\mu}_{u}+2 \mu_{s}\right)+\frac{1}{2} \bar{\mu}_{u} t_{3},
\end{aligned}
$$

where in the last line we have replaced $\mu_{u}$ and $\mu_{d}$ by $\bar{\mu}_{u}$. In practice the difference between $\mu_{u}, \mu_{d}$ and $\bar{\mu}_{u}$ is smaller than $10 \%$.

The effect of the baryon wave function renormalization due to the meson cloud represented by the diagrams (a) and (b), can be absorbed in the renormalized coupling constant $f_{\pi N N}$ used in the CBM [15].

\section{RESULTS}

Before presenting the results, we recall that the contributions from the valence quark core are given by the covariant spectator quark model as discussed in Sec. III. The formalism was discussed in detail in Ref. 35]. The important point to recall is that for $Q^{2}=0$ the valence quark contributions $G_{M}^{B}$ depend only on the quark anomalous moments $\kappa_{u}, \kappa_{d}, \kappa_{s}$ and the octet/decuplet radial wave functions through $\mathcal{I}(0)$. The corresponding parameters were fixed in the previous works.

We start to present the results by discussing the pion cloud contributions for the $\gamma^{*} N \rightarrow \Delta$ reaction, and explain how the meson cloud contributions are calibrated 


\begin{tabular}{|c|c|c|c|c|c|}
\hline & $G_{M}^{B}(0)$ & $G_{M}^{\mathrm{MCa}}(0)$ & $G_{M}^{\mathrm{MCb}}(0)$ & $G_{M}^{\mathrm{MC}}(0)$ & $G_{M}(0)$ \\
\hline \multirow[t]{4}{*}{$\gamma^{*} N \rightarrow \Delta$} & 1.633 & 0.713 & 0.610 & 1.323 & 2.956 \\
\hline & & 0.017 & 0.037 & & \\
\hline & & & 0.0062 & & \\
\hline & & 0.730 & 0.652 & 1.383 & 3.016 \\
\hline \multirow[t]{4}{*}{$\gamma^{*} \Lambda \rightarrow \Sigma^{* 0}$} & 1.683 & 0.669 & 0.358 & 1.027 & 2.710 \\
\hline & & 0.068 & 0.289 & & \\
\hline & & & 0.016 & & \\
\hline & & 0.737 & 0.663 & 1.400 & 3.083 \\
\hline \multirow[t]{4}{*}{$\gamma^{*} \Sigma^{+} \rightarrow \Sigma^{*+}$} & 2.094 & 0.149 & 0.513 & 0.663 & 2.757 \\
\hline & & 0.155 & 0.269 & & \\
\hline & & & 0.043 & & \\
\hline & & 0.304 & 0.825 & 1.129 & 3.224 \\
\hline \multirow[t]{4}{*}{$\gamma^{*} \Sigma^{0} \rightarrow \Sigma^{* 0}$} & 0.969 & 0.000 & 0.270 & 0.270 & 1.239 \\
\hline & & 0.104 & 0.010 & & \\
\hline & & & 0.015 & & \\
\hline & & 0.104 & 0.387 & 0.490 & 1.460 \\
\hline \multirow[t]{4}{*}{$\gamma^{*} \Sigma^{-} \rightarrow \Sigma^{*-}$} & -0.156 & -0.149 & 0.026 & -0.124 & -0.279 \\
\hline & & 0.052 & -0.065 & & \\
\hline & & & -0.012 & & \\
\hline & & -0.097 & -0.052 & -0.149 & -0.305 \\
\hline \multirow[t]{4}{*}{$\gamma^{*} \Xi^{0} \rightarrow \Xi^{* 0}$} & 2.191 & 0.222 & 0.086 & 0.308 & 2.499 \\
\hline & & 0.187 & 0.519 & & \\
\hline & & & 0.086 & & \\
\hline & & 0.410 & 0.691 & 1.101 & 3.291 \\
\hline \multirow[t]{4}{*}{$\gamma^{*} \Xi^{-} \rightarrow \Xi^{*-}$} & -0.168 & -0.222 & 0.084 & -0.138 & -0.306 \\
\hline & & 0.038 & -0.108 & & \\
\hline & & & -0.0034 & & \\
\hline & & -0.185 & -0.028 & -0.213 & -0.380 \\
\hline
\end{tabular}

TABLE III: Meson cloud contributions for the octet to decuplet transition magnetic moments. In each group the first line indicates the pion cloud contributions, the second line the kaon cloud, the third line the eta cloud, and the fourth the sum of all meson cloud contributions (boldface). The column $G_{M}^{B}(0)$ presents the contributions from the valence quark core. The column $G_{M}^{\mathrm{MC}}(0)$ and $G_{M}(0)$ show respectively the total meson cloud contributions and the final results (both boldface). The results in the first line for $G_{M}^{\mathrm{MC}}(0)$ and $G_{M}(0)$ include only the pion cloud contributions.

by this reaction. Next, we will present the results for all meson cloud contributions for the $\gamma^{*} B \rightarrow B^{\prime}$ reactions, and discuss the final results of the form factor $G_{M}$, and decay widths $\Gamma$. Finally, we will compare our results with those existing in the literature.

\section{A. Pion cloud contributions for the $\gamma^{*} N \rightarrow \Delta$ reaction}

The results for the pion cloud contribution arising from the diagrams (a) and (b), from the CBM, are presented in Table II (entry CBM). As we can see in Table II, the final contribution from the pion cloud in this case is 1.634, which is larger than the estimate made by the covariant spectator quark model of 1.323 by about $33 \%$ (see entry Spectator). This is not surprising, since the CBM tends 


\begin{tabular}{lcccc}
\hline \hline & $G_{M}(0)$ & $\left|G_{M}(0)\right|_{\exp }$ & $\Gamma(\mathrm{keV})$ & $\Gamma_{\exp }(\mathrm{keV})$ \\
\hline$\Delta \rightarrow \gamma N$ & 3.02 & $3.04 \pm 0.11[31]$ & 648 & $660 \pm 47[31]$ \\
$\Sigma^{* 0} \rightarrow \gamma \Lambda$ & 3.08 & $3.35 \pm 0.57[31]$ & 399 & $470 \pm 160[31]$ \\
& & $3.26 \pm 0.37[33,34]$ & & $445 \pm 102[33,34]$ \\
$\Sigma^{*+} \rightarrow \gamma \Sigma^{+}$ & 3.22 & $4.10 \pm 0.57[33]$ & 154 & $250 \pm 70[33]$ \\
$\Sigma^{* 0} \rightarrow \gamma \Sigma^{0}$ & 1.46 & & 32 & \\
$\Sigma^{*-} \rightarrow \gamma \Sigma^{-}$ & -0.31 & $<0.8[55]$ & 1.4 & $<9.5[55]$ \\
$\Xi^{* 0} \rightarrow \gamma \Xi^{0}$ & 3.29 & & 182 & \\
$\Xi^{*-} \rightarrow \gamma \Xi^{-}$ & -0.38 & & 2.4 & \\
\hline \hline
\end{tabular}

TABLE IV: Results for $G_{M}(0)$ corresponding to the $B^{\prime} \rightarrow \gamma B$ decays. The values for $\left|G_{M}(0)\right|_{\exp }$ are estimated by Eq. (5.5) using the experimental values of $\Gamma_{B^{\prime} \rightarrow \gamma B}$.

to overestimate the effect of the pion cloud for the $\gamma^{*} N \rightarrow$ $\Delta$ reaction. Indeed, in Ref. [15] the pion cloud gives a contribution of about $66 \%$ of the total, a contribution substantially larger than in the other calculations [1, 27, 50].

On the other hand, the pion cloud contribution in the covariant spectator quark model were determined by a fit to the $\gamma^{*} N \rightarrow \Delta$ data for $Q^{2} \leq 6 \mathrm{GeV}^{2}$, combined with the estimate made for the quark core contribution extracted by the Excited Baryon Analysis Center model [50] by removing the meson cloud contributions. In addition, the estimate of the quark core contributions from the covariant spectator quark model was compared successfully with the results of lattice QCD simulations using the pion masses around $350-650 \mathrm{MeV}$, where the pion cloud contribution is expected to be suppressed. To compare with the lattice QCD data, the model was generalized to the lattice QCD regime using the vector meson dominance parametrization for the quark current. See details in Refs. 29, 30, 39, 40]. All these results show that the covariant spectator quark model provides a robust description of both the physical and lattice QCD data, and that it is probably more appropriated than the CBM parametrization for the present study. The small deviation from the result for $Q^{2}=0$ (bare plus pion cloud) given by the covariant quark model, 2.96, compared to the experimental result of $3.02 \pm 0.03$ [54], is a consequence of the global fit of the covariant spectator quark model for $Q^{2} \leq 6 \mathrm{GeV}^{2}$, instead of fitting only to the low $Q^{2}$ region data.

In the following we will use $\tilde{G}_{M}$ to represent the CBM result, and $G_{M}$ for the present model (covariant spectator quark model). Also to distinguish between the different $\gamma^{*} B \rightarrow B^{\prime}$ reactions, we will use the argument $B B^{\prime}$ as $G_{M}\left(B B^{\prime}\right)$. Recall that we are only discussing the form factors at $Q^{2}=0$.

\section{B. Meson cloud contributions for the $\gamma^{*} B \rightarrow B^{\prime}$ reactions}

In order to keep the successful features of the pion cloud contributions estimated in the covariant spectator quark model for the $\gamma^{*} N \rightarrow \Delta$ transition, we normalize the CBM result for the pion cloud $\tilde{G}_{M}^{\mathrm{MC} \pi}(0)$ by the result of the covariant spectator quark model $G_{M}^{\mathrm{MC} \pi}(0)=3 \lambda_{\pi}=$ 1.32 , given by,

$$
G_{M}^{\mathrm{MC} \pi}(N \Delta)=\mathcal{R} \tilde{G}_{M}^{\mathrm{MC} \pi}(N \Delta),
$$

where

$$
\mathcal{R}=\frac{3 \lambda_{\pi}}{\tilde{G}_{M}^{\mathrm{MC} \pi}(N \Delta)} .
$$

Numerically it gives $\mathcal{R} \simeq 0.81$.

To estimate the effect of the other meson clouds, the kaon and $\eta$ meson in the $\gamma^{*} N \rightarrow \Delta$ reaction, and also for the other octet to decuplet transitions, we use a similar relation, since all the couplings are related with the coupling constant $f_{\pi N N}$. Thus, we use in general,

$$
G_{M}^{\mathrm{MC}}\left(B B^{\prime}\right)=\mathcal{R} \tilde{G}_{M}^{\mathrm{MC}}\left(B B^{\prime}\right)
$$

Except for the fact that we now include the diagram (b), the procedure is the same as the one used in the previous work [35]. From Eq. (2.4), we get

$$
f_{B B^{\prime}}=\frac{\tilde{G}_{M}^{\mathrm{MC}}\left(B B^{\prime}\right)}{\tilde{G}_{M}^{\mathrm{MC} \pi}(N \Delta)} .
$$

The results of the meson cloud contributions from the diagrams (a) and (b) for the octet to decuplet electromagnetic transition form factors at $Q^{2}=0$, are presented in Table III Since we expect the results for $\gamma^{*} n \rightarrow \Delta^{0}$ and $\gamma^{*} p \rightarrow \Delta^{+}$to be the same in the present approach, we use the label $\gamma^{*} N \rightarrow \Delta$ to represent both reactions.

In Table III we can see the contributions from each meson, $\pi, K$ or $\eta$. We can conclude that the pion cloud indeed gives the dominant meson cloud contribution for 
the $\gamma^{*} N \rightarrow \Delta$ reaction. However, for the other reactions, particularly the kaon cloud, can give important contributions. The magnitude of the kaon plus eta cloud contributions can be obtained by subtracting the result of the first line (only pion cloud effects) from the last line (bold, total) for $G_{M}(0)$. We can then conclude that the kaon and eta cloud corrections are about 0.4 for $\gamma^{*} \Lambda \rightarrow \Sigma^{* 0}$ and 0.5 for $\gamma^{*} \Sigma^{+} \rightarrow \Sigma^{*+}$.

The kaon cloud effects in some cases are comparable, or larger than those of the pion cloud, particularly for the diagram (b). See for instance the reactions $\gamma^{*} \Lambda \rightarrow \Sigma^{* 0}$, $\gamma^{*} \Sigma^{+} \rightarrow \Sigma^{*+}$ and $\gamma^{*} \Xi^{0} \rightarrow \Xi^{* 0}$.

Globally, the meson cloud contributions can be about $45 \%$ of the total for the cases with $\left|G_{M}(0)\right| \approx 3$, or even larger for the $\gamma^{*} \Sigma^{*-} \rightarrow \Sigma^{-}$and $\gamma^{*} \Xi^{*-} \rightarrow \Xi^{-}$cases.

Another interesting point is the magnitude of the contributions from the diagram (b). They are in most cases similar or larger than the contributions from the diagram (a). This is a consequence of two main factors: i) the quark magnetic moments $\mu_{q}$ are significant (about 2-3 nuclear magneton), which enhances the effect; ii) the diagram (b) has a large number of intermediate states to be summed over. See Appendix A.

Note that, this feature contradicts the assumption made in the previous work [35], that the diagram (a) is expected to give the leading order contribution. However, since in the previous study the meson cloud contributions were normalized by the total pion cloud contribution of the $\gamma^{*} N \rightarrow \Delta$ transition, the difference between the previous and the new results for all the reactions is not drastic. Based on the present results for the $\gamma^{*} N \rightarrow \Delta$ transition, where roughly $50 \%$ of the meson cloud comes from diagram (a) and (b), we may regard the previous result as a consequence of the assumption that both diagrams have the same effect $(50 \%)$ for all the reactions. Recall that only the pion was considered in the previous work.

In the present study, we also normalize the meson cloud contributions by the pion cloud contribution for the $\gamma^{*} N \rightarrow \Delta$ transition, but we leave the contributions from the diagrams (a) and (b) independent, as can be seen in Table III

Then, we can conclude that the explicit inclusion of the contributions form the diagram (b), increases the contribution of the meson cloud, and improves the description of the $\Sigma^{* 0} \rightarrow \gamma \Lambda$ and $\Sigma^{*+} \rightarrow \gamma \Sigma^{+}$data as will be discussed next. For further discussion, we recall that the $\Sigma^{* 0} \rightarrow \gamma \Lambda$ and $\Sigma^{*+} \rightarrow \gamma \Sigma^{+}$decay widths given in the Particle Data Group (PDG) 31], were underestimated respectively 1.2 and 2.4 standard deviations in the previous work [35].

The final results for $G_{M}(0)$ are also presented in Table IV] in comparison with the estimates extracted from the experimental decay widths 35]. The estimates were made assuming the dominance of $G_{M}(0)$ compared to the quadrupole electric form factor $G_{E}(0)$. From Table IV one can see that the present model can describe well the data for the $\Sigma^{* 0} \rightarrow \gamma \Lambda$ (less than one standard deviation), and underestimates the $\Sigma^{*+} \rightarrow \gamma \Sigma^{+}$data 1.5 standard deviations. These features may be regarded as a significant improvement compared to our previous result and other theoretical estimates (see discussion in the next section).

Using the model results obtained for $G_{M}(0)$, we calculate the decuplet electromagnetic decay widths, assuming the dominance of $G_{M}$,

$$
\Gamma_{B^{\prime} \rightarrow \gamma B}=\frac{\alpha}{16} \frac{\left(M_{B^{\prime}}^{2}-M_{B}^{2}\right)^{3}}{M_{B^{\prime}}^{3} M_{B}^{2}}\left|G_{M}(0)\right|^{2},
$$

where $\alpha=\frac{e^{2}}{4 \pi} \simeq \frac{1}{137}$ is the electromagnetic fine structure constant. The $G_{M}$ dominance is a good approximation according to theoretical estimates and the experimental results for the $\gamma^{*} N \rightarrow \Delta$. (See Ref. [35] for a more detailed discussion.)

Our predictions for the decay width, $\Gamma \equiv \Gamma_{B^{\prime} \rightarrow \gamma B}$, are also presented in Table [V] For the cases of $\Delta \rightarrow$ $\gamma N$ and $\Sigma^{* 0} \rightarrow \gamma \Lambda$, we also present the results from PDG [31]. In addition we present the results for $\Sigma^{* 0} \rightarrow$ $\gamma \Lambda$, and $\Sigma^{*+} \rightarrow \gamma \Sigma^{+}$from Refs. [33, 34]. Our model results deviate from the data only for the $\Sigma^{*+} \rightarrow \gamma \Sigma^{+}$ reaction by 1.4 standards deviations. The upper limit for the $\Sigma^{*-} \rightarrow \gamma \Sigma^{-}$reaction [55], is also shown in Table IV]

We call attention to the fact that the present estimate of the meson cloud contribution is affected by some uncertainties related to the effective quark magnetic moments used to calculate the diagram (b). Since some of the intermediate states correspond to elastic transitions (where $\mathcal{I}(0)=1$ ), we can question the use of the prescription (3.9) with the factor $\mathcal{I}(0) \leq 1$ (octet-decuplet radial wave function overlap integral), given by the inelastic $\gamma^{*} B \rightarrow B^{\prime}$ reaction. Therefore, an upper limit for the meson cloud contribution can be obtained by setting $\mathcal{I}(0)=1$ (perfect overlap of the radial wave functions). In this case the final results for the decay width are enhanced by $1-6 \%$. In particular, the $\Sigma^{* 0} \rightarrow \gamma \Lambda$ decay width increases by $4.4 \%$ and that for the $\Sigma^{*+} \rightarrow \gamma \Sigma^{+}$ case in $2.6 \%$. Therefore, in the latter case, a possible enhancement due to the intermediate state baryon wave function overlaps is small, and it does not significantly increase the final result to enough to bring the present result closer to the experimental data.

Taking into account the typical uncertainty in the CBM of about $10 \%$, and also assuming that the meson cloud contribution is about $50 \%$ of the total in the CBM, this gives about $5 \%$ ambiguity. Combining the two ambiguities, one from the wave function overlap of $1-6 \%$, and the other from the CBM estimate for meson cloud contributions of about $5 \%$, we can conclude that our estimate can be affected by a value around $10 \%$.

Another interesting exercise can be to check if the discrepancy between our estimate and the experimental $\Sigma^{*+} \rightarrow \gamma \Sigma^{+}$decay width, may be a consequence of neglecting the effect of $G_{E}(0)$ in the calculation of the decay width. In this case, the deviation from the data would be the result of dropping the term $3\left|G_{E}(0)\right|^{2}$ in the sum with $\left|G_{M}(0)\right|^{2}$ in the decay width calculation. Using our 


\begin{tabular}{lccc}
\hline \hline & $\Delta \rightarrow \gamma N$ & $\Sigma^{* 0} \rightarrow \gamma \Lambda$ & $\Sigma^{*+} \rightarrow \gamma \Sigma^{+}$ \\
\hline U-spin [7, 35] & & $292 \pm 27$ & $138 \pm 13$ \\
HB $\chi$ PT [23] & $670-790$ & $252-540$ & $70-220$ \\
Alg. Mod. [13] & $342-344$ & 221.3 & 140.7 \\
QCD SR [22] & 887 & 409 & 150 \\
Large $N_{c}[20]$ & $669 \pm 42$ & $336 \pm 81$ & $149 \pm 36$ \\
Spectator & 648 & 399 & 154 \\
Data [31, 33, 34] & $660 \pm 47$ & $470 \pm 160$ & $250 \pm 70$ \\
& & $445 \pm 102$ & \\
\hline \hline
\end{tabular}

TABLE V: Results for the $B^{\prime} \rightarrow \gamma B$ decay widths (in keV) given by several works.

result for $\left|G_{M}(0)\right|$, we would be able to reproduce the experimental $\Sigma^{*+} \rightarrow \gamma \Sigma^{+}$decay width if $\left|G_{E}(0)\right|$ is about $30 \%$ of $\left|G_{M}(0)\right|$.

\section{Discussion}

In general, most of the existing quark models underestimate the $\Sigma^{* 0} \rightarrow \gamma \Lambda$ and $\Sigma^{*+} \rightarrow \gamma \Sigma^{+}$decay widths by about $50 \%$. Chiral quark models with mesonic effects are lso included in this category [12, 16, 17]. In those cases they give a $\Delta \rightarrow \gamma N$ decay width of about $400 \mathrm{keV}$, and smaller than the experimental result of $660 \pm 47 \mathrm{keV}$. A more detailed comparison between the model results and data can be found in the previous work [35].

A better result for the $\Sigma^{*+} \rightarrow \gamma \Sigma^{+}$decay width is obtained by an algebraic model of the hadronic structure 13]. However, the $\Delta$ decay width is again underestimated (see Table V).

Some calculations give closer values to the experimental results, e.g., the Heavy Baryon Chiral Perturbation Theory [23] as presented in Table V]. The windows associated with the results are however, too broad.

Also, the predictions based on U-spin symmetry proposed in the seventies [7], give a good description of the data, using the updated result for the $\Delta \rightarrow \gamma N$ decay width 35]. As can be seen in Table $\mathrm{V}$ the best result differs, respectively, by 0.8 and 1.4 standard deviations for the $\Lambda$ and $\Sigma^{+}$cases. It is worth mentioning that the estimates made in Ref. [33], also based on a U-spin symmetry, have a much better agreement with the data. However, as also discussed in our previous work 35], their U-spin symmetry-based estimates did not take into account the effect of the baryon masses in the conversion between the form factors and the helicity amplitudes.

The results from QCD sum rules [22] are close to the $\Sigma^{*+} \rightarrow \gamma \Sigma^{+}$and $\Sigma^{* 0} \rightarrow \gamma \Lambda$ decay width data, but overestimate the $\Delta \rightarrow \gamma N$ decay width by about $220 \mathrm{keV}$.

An excellent description of all the data was also obtained in Ref. [20] using a $1 / N_{c}$ expansion. The unknown coefficients in the expansion are fitted to the known octet and decuplet magnetic moments $\left(n, p, \Sigma^{ \pm}, \Xi^{0,-}, \Delta^{+}, \Omega^{-}\right.$ and $\Sigma^{0} \rightarrow \gamma \Lambda$ transition), providing a prediction for the remaining cases. Note that the values from Ref. [20] are very close to our own results.

We would like to emphasize that our estimate is a pure prediction, since the parameters involved in the calculations (quarks anomalous magnetic moments and wave functions) were already determined and calibrated in the previous works. The only adjustable ingredient in the present calculation is the magnitude of the pion cloud contribution for the $\gamma^{*} N \rightarrow \Delta$ reaction, chosen to match the pion cloud contribution of the original covariant spectator quark model [28].

\section{CONCLUSIONS}

In this work we have studied the decuplet to octet electromagnetic decay widths, which are related to the magnetic transition form factors defined at $Q^{2}=0$. To describe the baryon quark core we have used the covariant spectator quark model, the model parameters of which are calibrated in the previous works on the octet and decuplet baryon systems. To estimate the effects of the meson cloud, including those from the pion, kaon and eta meson, we have been guided by the cloudy bag model, improved by the result from the covariant spectator quark model for the $\gamma^{*} N \rightarrow \Delta$ reaction. The effects included as the meson cloud are, the direct photon coupling to the meson (diagram (a)), and the photon coupling to the intermediate baryon states while one meson is in the air (diagram (b)).

We conclude that the inclusion of the contributions from the diagram (b), as well as the effects of the kaon cloud (diagrams (a) and (b)), are both very important. When the meson cloud contributions are combined with the quark core contributions calculated by the covariant spectator quark model, the present model can reproduce the experimental results well. The inclusion of only the valence quark contributions, leads to significant underestimates of the data. The meson cloud effects are particularly important for the reactions $\Sigma^{* 0} \rightarrow \gamma \Lambda$ and $\Sigma^{*+} \rightarrow \gamma \Sigma^{+}$. Furthermore, the effect of the diagram (b) is also very important for the $\Delta \rightarrow \gamma N$ reaction.

In summary, we are able to describe the $\Sigma^{* 0} \rightarrow \gamma \Lambda$ 
decay width very well, and also obtain a very reasonable result (1.4 standard deviations) for that of the $\Sigma^{*+} \rightarrow$ $\gamma \Sigma^{+}$. The present approach also describes the $\Delta \rightarrow \gamma N$ decay width rather well. However, in the last case, the agreement is a consequence of the fit made previously by the model, although the explicit inclusion of the extra kaon cloud effects improves the agreement slightly.

Our predictions for the transition form factors of the other reactions are consistent with the estimates made based on the U-spin symmetry, namely, $G_{M}\left(\Sigma^{*+} \Sigma^{+}\right) \approx$ $G_{M}\left(\Xi^{* 0} \Xi^{0}\right)$, and $G_{M}\left(\Sigma^{*-} \Sigma^{-}\right) \approx G_{M}\left(\Xi^{*-} \Xi^{-}\right)$.

We can, in general, conclude that the meson cloud effects are of fundamental importance to describe the $\gamma^{*} B \rightarrow B^{\prime}$ reactions, especially in the low $Q^{2}$ region, and the decuplet baryon decay widths. To test further the conclusions of the present study, accurate experimental determination of the unknown decuplet baryon electromagnetic decay widths is crucial. In addition, precise lattice QCD simulations for several pion mass values can also help to constrain the contributions form the valence quarks, and test our estimates of the quark core contributions.

\section{Acknowledgments}

The authors would like to thank Y. Kohyama for the CBM note, which helped the present study. This work was supported by the Brazilian Ministry of Science, Technology and Innovation (MCTI-Brazil), and Conselho Nacional de Desenvolvimento Científico e Tecnológico (CNPq), project 550026/2011-8.

\section{Appendix A: Meson cloud contributions}

The meson cloud contributions calculated by the CBM corresponding to the diagram (a) are presented in Table VI. Compared to the results presented in Ref. 35] the present results include a factor $\sqrt{\frac{2}{3}}\left(2 M_{B}\right)$ in each transition. The factor is necessary to represent the form factors in its natural units (dimensionless). Note that, the factor $\left(2 M_{B}\right)$ multiplied by $H_{B B^{\prime}}^{M}\left(B_{1}\right)$ gives a dimensionless quantity.

In the exact $\mathrm{SU}(3)$ limit, the factors $\sqrt{\frac{2}{3}}\left(2 M_{B}\right)$ become the same for all the octet to decuplet transitions, and as a consequence, the factor can be ignored in the calculation of $f_{B B^{\prime}}$, since the factors will be canceled out by the normalization, divided by the $\gamma^{*} N \rightarrow \Delta$ contribution following the procedure of this work. Taking the limit $H_{B B^{\prime}}^{\pi}\left(B_{1}\right)=H_{\pi}$ (independent of the octet and decuplet baryon masses) and $H_{B B^{\prime}}^{K}\left(B_{1}\right)=0$, we recover the previous results given in Ref. [35] for the contributions from the diagram (a).

To calculate the contributions from the diagram (b) in
Fig. 1] it is convenient to define the following quantities:

$$
\begin{aligned}
& \mu_{S}=\frac{1}{3}\left(\bar{\mu}_{u}+2 \mu_{s}\right), \\
& \mu_{V}=\bar{\mu}_{u}, \\
& \mu_{1}=\frac{1}{3}\left(2 \bar{\mu}_{u}+\mu_{s}\right), \\
& \mu_{2}=\bar{\mu}_{u}-\mu_{s}, \\
& \mu_{3}=\frac{1}{9}\left(\bar{\mu}_{u}+8 \mu_{s}\right), \\
& \mu_{4}=\frac{1}{3}\left(-\bar{\mu}_{u}+4 \mu_{s}\right) .
\end{aligned}
$$

Note that the quantities above are dependent on the transitions under consideration [see Eq. (3.9)].

We can now write the meson cloud contributions corresponding to the diagram (b) as:

$$
\begin{aligned}
& \tilde{G}_{M}^{\mathrm{MCb}}(N \Delta)=\frac{2 \sqrt{2}}{3} \mu_{V} \\
& \times\left\{\frac{4}{9} H_{N \Delta}^{2 \pi}(N, N)+\frac{5}{9} H_{N \Delta}^{2 \pi}(N, \Delta)+\frac{8}{225} H_{N \Delta}^{2 \pi}(\Delta, N)\right. \\
& +\frac{4}{9} H_{N \Delta}^{2 \pi}(\Delta, \Delta)+\frac{4}{25} H_{N \Delta}^{2 K}(\Lambda, \Sigma)+\frac{1}{5} H_{N \Delta}^{2 K}\left(\Lambda, \Sigma^{*}\right) \\
& +\frac{8}{225} H_{N \Delta}^{2 K}(\Sigma, \Sigma)+\frac{4}{45} H_{N \Delta}^{2 K}\left(\Sigma^{*}, \Sigma^{*}\right)-\frac{1}{45} H_{N \Delta}^{2 K}\left(\Sigma, \Sigma^{*}\right) \\
& \left.+\frac{4}{225} H_{N \Delta}^{2 K}\left(\Sigma^{*}, \Sigma\right)+\frac{1}{15} H_{N \Delta}^{2 \eta}(N, \Delta)\right\},
\end{aligned}
$$

$$
\begin{aligned}
\tilde{G}_{M}^{\mathrm{MCb}}\left(\Lambda \Sigma^{* 0}\right)=\sqrt{\frac{2}{3}} \frac{M_{\Lambda}}{M_{N}} \mu_{V} \\
\times\left\{\frac{8}{75} H_{\Lambda \Sigma^{*}}^{2 \pi}(\Sigma, \Lambda)+\frac{32}{225} H_{\Lambda \Sigma^{*}}^{2 \pi}(\Sigma, \Sigma)+\frac{8}{45} H_{\Lambda \Sigma^{*}}^{2 \pi}\left(\Sigma, \Sigma^{*}\right)\right. \\
\quad+\frac{4}{75} H_{\Lambda \Sigma^{*}}^{2 \pi}\left(\Sigma^{*}, \Lambda\right)-\frac{8}{225} H_{\Lambda \Sigma^{*}}^{2 \pi}\left(\Sigma^{*}, \Sigma\right)+\frac{16}{45} H_{\Lambda \Sigma^{*}}^{2 \pi}\left(\Sigma^{*}, \Sigma^{*}\right) \\
+\frac{4}{15} H_{\Lambda \Sigma^{*}}^{2 K}(N, N)+\frac{8}{15} H_{\Lambda \Sigma^{*}}^{2 K}(N, \Delta)+\frac{4}{225} H_{\Lambda \Sigma^{*}}^{2 K}(\Xi, \Xi) \\
\quad+\frac{4}{45} H_{\Lambda \Sigma^{*}}^{2 K}\left(\Xi, \Xi^{*}\right)+\frac{8}{225} H_{\Lambda \Sigma^{*}}^{2 K}\left(\Xi^{*}, \Xi\right)+\frac{8}{45} H_{\Lambda \Sigma^{*}}^{2 K}\left(\Xi^{*}, \Xi^{*}\right) \\
\left.\quad+\frac{8}{75} H_{\Lambda \Sigma^{*}}^{2 \eta}(\Lambda, \Sigma)\right\}
\end{aligned}
$$




\begin{tabular}{lc}
\hline \hline$\gamma^{*} N \rightarrow \Delta$ & $\tilde{G}_{M}^{\mathrm{MCa}}\left(B B^{\prime}\right)$ \\
$\gamma^{*} \Lambda \rightarrow \Sigma^{*}$ & $\tilde{G}_{M}^{\mathrm{MCa}}\left(\Lambda \Sigma^{*}\right)=\frac{2 \sqrt{2}}{15 \sqrt{3}}\left(2 M_{\Lambda}\right)\left[\frac{4}{5} H_{\Lambda \Sigma^{*}}^{\pi}(\Sigma)+4 H_{\Lambda \Sigma^{*}}^{\pi}\left(\Sigma^{*}\right)+\frac{3}{5} H_{\Lambda \Sigma^{*}}^{K}(N)-\frac{1}{5} H_{\Lambda \Sigma^{*}}^{K}(\Xi)+2 H_{\Lambda \Sigma^{*}}^{K}\left(\Xi^{*}\right)\right]$ \\
$\gamma^{*} \Sigma \rightarrow \Sigma^{*}$ & $\tilde{G}_{M}^{\mathrm{MCa}}\left(\Sigma \Sigma^{*}\right)=\frac{\sqrt{2}}{3}\left(2 M_{\Sigma}\right)\left[\frac{2}{75} H_{\Sigma \Sigma^{*}}^{K}(N)+\frac{8}{15} H_{\Sigma \Sigma^{*}}^{K}(\Delta)+\frac{2}{15} H_{\Sigma \Sigma^{*}}^{K}(\Xi)+\frac{4}{15} H_{\Sigma \Sigma^{*}}^{K}\left(\Xi^{*}\right)\right]+$ \\
& $\frac{\sqrt{2}}{3}\left(2 M_{\Sigma}\right)\left[\frac{4}{25} H_{\Sigma \Sigma^{*}}^{\pi}(\Lambda)-\frac{8}{75} H_{\Sigma \Sigma^{*}}^{\pi}(\Sigma)+\frac{4}{15} H_{\Sigma \Sigma^{*}}^{\pi}\left(\Sigma^{*}\right)\right.$ \\
& $\left.-\frac{2}{75} H_{\Sigma \Sigma^{*}}^{K}(N)+\frac{4}{15} H_{\Sigma \Sigma^{*}}^{K}(\Delta)+\frac{2}{15} H_{\Sigma \Sigma^{*}}^{K}(\Xi)+\frac{4}{15} H_{\Sigma \Sigma^{*}}^{K}\left(\Xi^{*}\right)\right] J_{3}$ \\
$\gamma^{*} \Xi \rightarrow \Xi^{*}$ & $\tilde{G}_{M}^{\mathrm{MCa}}\left(\Xi \Xi^{*}\right)=\frac{\sqrt{2}}{3}\left(2 M_{\Xi}\right)\left[-\frac{1}{25} H_{\Xi \Xi^{*}}^{K}(\Lambda)+\frac{1}{5} H_{\Xi \Xi^{*}}^{K}(\Sigma)+\frac{2}{5} H_{\Xi \Xi^{*}}^{K}\left(\Sigma^{*}\right)+\frac{2}{5} H_{\Xi \Xi^{*}}^{K}(\Omega)\right]+$ \\
& $\frac{\sqrt{2}}{3}\left(2 M_{\Xi}\right)\left[\frac{4}{75} H_{\Xi \Xi^{*}}^{\pi}(\Xi)+\frac{4}{15} H_{\Xi \Xi^{*}}^{\pi}\left(\Xi^{*}\right)+\frac{1}{25} H_{\Xi \Xi^{*}}^{K}(\Lambda)+\frac{1}{15} H_{\Xi \Xi^{*}}^{K}(\Sigma)+\frac{2}{15} H_{\Xi \Xi^{*}}^{K}\left(\Sigma^{*}\right)+\frac{2}{5} H_{\Xi \Xi^{*}}^{K}(\Omega)\right] \tau_{3}$ \\
\hline \hline
\end{tabular}

TABLE VI: Meson cloud contributions for $G_{M}$ from the diagram (a) in Fig. 1

$$
\begin{aligned}
& \tilde{G}_{M}^{\mathrm{MCb}}\left(\Sigma \Sigma^{*}\right)=\frac{\sqrt{2}}{3} \frac{M_{\Sigma}}{M_{N}} \\
& \times\left\{\mu _ { S } \left[\frac{16}{45} H_{\Sigma \Sigma^{*}}^{2 \pi}\left(\Sigma, \Sigma^{*}\right)+\frac{8}{225} H_{\Sigma \Sigma^{*}}^{2 \pi}\left(\Sigma^{*}, \Sigma\right)\right.\right. \\
& \left.+\frac{4}{75} H_{\Sigma \Sigma^{*}}^{2 \eta}\left(\Sigma^{*}, \Sigma\right)+\frac{4}{9} H_{\Sigma \Sigma^{*}}^{2 K}\left(\Xi, \Xi^{*}\right)-\frac{8}{225} H_{\Sigma \Sigma^{*}}^{2 K}\left(\Xi^{*}, \Xi\right)\right] \\
& +\mu_{1}\left[\frac{32}{225} H_{\Sigma \Sigma^{*}}^{2 \pi}(\Sigma, \Sigma)+\frac{8}{75} H_{\Sigma \Sigma^{*}}^{2 \eta}(\Sigma, \Sigma)\right] \\
& +\mu_{s} \frac{8}{75} H_{\Sigma \Sigma^{*}}^{2 \pi}(\Lambda, \Lambda)-\mu_{2} \frac{16}{135} H_{\Sigma \Sigma^{*}}^{2 \pi}\left(\Sigma^{*}, \Sigma^{*}\right) \\
& +\mu_{V}\left[\frac{4}{225} H_{\Sigma \Sigma^{*}}^{2 K}(N, N)+\frac{16}{45} H_{\Sigma \Sigma^{*}}^{2 K}(\Delta, \Delta)\right] \\
& \left.+\mu_{3} \frac{4}{15} H_{\Sigma \Sigma^{*}}^{2 K}(\Xi, \Xi)+\mu_{4} \frac{8}{45} H_{\Sigma \Sigma^{*}}^{2 K}\left(\Xi^{*}, \Xi^{*}\right)\right\} \\
& +J_{3} \frac{\sqrt{2}}{3} \frac{M_{\Sigma}}{M_{N}} \mu_{V} \\
& \times\left\{-\frac{8}{75} H_{\Sigma \Sigma^{*}}^{2 \pi}(\Lambda, \Sigma)+\frac{4}{15} H_{\Sigma \Sigma^{*}}^{2 \pi}\left(\Lambda, \Sigma^{*}\right)\right. \\
& +\frac{16}{75} H_{\Sigma \Sigma^{*}}^{2 \pi}(\Sigma, \Lambda)+\frac{32}{225} H_{\Sigma \Sigma^{*}}^{2 \pi}(\Sigma, \Sigma)+\frac{8}{45} H_{\Sigma \Sigma^{*}}^{2 \pi}\left(\Sigma, \Sigma^{*}\right) \\
& -\frac{4}{75} H_{\Sigma \Sigma^{*}}^{2 \pi}\left(\Sigma^{*}, \Lambda\right)+\frac{4}{225} H_{\Sigma, \Sigma^{*}}^{2 \pi}\left(\Sigma^{*}, \Sigma\right)-\frac{8}{45} H_{\Sigma, \Sigma^{*}}^{2 \pi}\left(\Sigma^{*}, \Sigma^{*}\right) \\
& +\frac{4}{45} H_{\Sigma \Sigma^{*}}^{2 K}(N, N)-\frac{4}{45} H_{\Sigma \Sigma^{*}}^{2 K}(N, \Delta)+\frac{16}{225} H_{\Sigma \Sigma^{*}}^{2 K}(\Delta, N) \\
& +\frac{8}{9} H_{\Sigma \Sigma^{*}}^{2 K}(\Delta, \Delta)+\frac{4}{45} H_{\Sigma \Sigma^{*}}^{2 K}(\Xi, \Xi)+\frac{4}{9} H_{\Sigma \Sigma^{*}}^{2 K}\left(\Xi, \Xi^{*}\right) \\
& -\frac{8}{225} H_{\Sigma \Sigma^{*}}^{2 K}\left(\Xi^{*}, \Xi\right)-\frac{8}{45} H_{\Sigma \Sigma^{*}}^{2 K}\left(\Xi^{*}, \Xi^{*}\right) \\
& \left.+\frac{16}{75} H_{\Sigma \Sigma^{*}}^{2 \eta}(\Sigma, \Sigma)+\frac{4}{75} H_{\Sigma \Sigma^{*}}^{2 \eta}\left(\Sigma^{*}, \Sigma\right)\right\},
\end{aligned}
$$

$$
\begin{aligned}
& \tilde{G}_{M}^{\mathrm{MCb}}\left(\Xi \Xi^{*}\right)=\frac{\sqrt{2}}{3} \frac{M_{\Xi}}{M_{N}} \\
& \times\left\{\mu _ { S } \left[-\frac{1}{15} H_{\Xi \Xi^{*}}^{2 \pi}\left(\Xi, \Xi^{*}\right)+\frac{4}{75} H_{\Xi \Xi^{*}}^{2 \pi}\left(\Xi^{*}, \Xi\right)+\frac{1}{5} H_{\Xi \Xi^{*}}^{2 \eta}\left(\Xi, \Xi^{*}\right)\right.\right. \\
&+\left.\frac{4}{75} H_{\Xi \Xi^{*}}^{2 \eta}\left(\Xi^{*}, \Xi\right)+\frac{2}{3} H_{\Xi \Xi^{*}}^{2 K}\left(\Sigma, \Sigma^{*}\right)-\frac{4}{75} H_{\Xi \Xi^{*}}^{2 K}\left(\Sigma^{*}, \Sigma\right)\right] \\
&+ \mu_{3}\left[+\frac{2}{25} H_{\Xi \Xi^{*}}^{2 \pi}(\Xi, \Xi)+\frac{6}{25} H_{\Xi \Xi^{*}}^{2 \eta}(\Xi, \Xi)\right] \\
&+ \mu_{4}\left[\frac{2}{15} H_{\Xi \Xi^{*}}^{2 \pi}\left(\Xi^{*}, \Xi^{*}\right)-\frac{2}{15} H_{\Xi \Xi^{*}}^{2 \eta}\left(\Xi^{*}, \Xi^{*}\right)\right]+\mu_{1} \frac{4}{15} H_{\Xi \Xi^{*}}^{2 K}(\Sigma, \Sigma) \\
&+\left.\mu_{s}\left[\frac{4}{75} H_{\Xi \Xi^{*}}^{2 K}(\Lambda, \Lambda)+\frac{8}{15} H_{\Xi \Xi^{*}}^{2 K}(\Omega, \Omega)\right]+\mu_{2} \frac{8}{45} H_{\Xi \Xi^{*}}^{2 K}\left(\Sigma^{*}, \Sigma^{*}\right)\right\} \\
&+ \tau_{3} \frac{\sqrt{2}}{3} \frac{M_{\Xi}}{M_{N}} \mu_{V}\left\{-\frac{2}{225} H_{\Xi \Xi^{*}}^{2 \pi}(\Xi, \Xi)+\frac{1}{45} H_{\Xi \Xi^{*}}^{2 \pi}\left(\Xi, \Xi^{*}\right)\right. \\
&+\frac{2}{45} H_{\Xi \Xi^{*}}^{2 \pi}\left(\Xi^{*}, \Xi^{*}\right)-\frac{4}{225} H_{\Xi \Xi^{*}}^{2 \pi}\left(\Xi^{*}, \Xi\right)-\frac{4}{75} H_{\Xi \Xi^{*}}^{2 K}(\Lambda, \Sigma) \\
&+\frac{2}{15} H_{\Xi \Xi^{*}}^{2 K}\left(\Lambda, \Sigma^{*}\right)+\frac{4}{15} H_{\Xi \Xi^{*}}^{2 K}(\Sigma, \Lambda)+\frac{16}{45} H_{\Xi \Xi^{*}}^{2 K}(\Sigma, \Sigma) \\
&+\frac{4}{9} H_{\Xi \Xi^{*}}^{2 K}\left(\Sigma, \Sigma^{*}\right)+\frac{4}{75} H_{\Xi \Xi^{*}}^{2 K}\left(\Sigma^{*}, \Lambda\right)-\frac{8}{225} H_{\Xi \Xi^{*}}^{2 K}\left(\Sigma^{*}, \Sigma\right) \\
&+\frac{16}{45} H_{\Xi \Xi^{*}}^{2 K}\left(\Sigma^{*}, \Sigma^{*}\right)+\frac{2}{25} H_{\Xi \Xi^{*}}^{2 \eta}(\Xi, \Xi)+\frac{1}{5} H_{\Xi \Xi^{*}}^{2 \eta}\left(\Xi, \Xi^{*}\right) \\
&\left.+\frac{4}{75} H_{\Xi \Xi^{*}}^{2 \eta}\left(\Xi^{*}, \Xi\right)+\frac{2}{15} H_{\Xi \Xi^{*}}^{2 \eta}\left(\Xi^{*}, \Xi^{*}\right)\right\} .
\end{aligned}
$$

In the equations above the factor $\frac{M_{B}}{M_{N}}$ is a consequence of the factor $\sqrt{\frac{2}{3}}\left(2 M_{B}\right)$ combined with $1 /\left(2 M_{N}\right)$ in units of the quark magnetic moments. The final result is thus dimensionless.
[1] V. D. Burkert and T. S. H. Lee, Int. J. Mod. Phys. E 13, 1035 (2004).

[2] I. G. Aznauryan, A. Bashir, V. Braun, S. J. Brodsky, V. D. Burkert, L. Chang, C. .Chen and B. El-
Bennich et al., Int. J. Mod. Phys. E 22, 1330015 (2013) arXiv:1212.4891 [nucl-th]].

[3] P. Geiger and N. Isgur, Phys. Rev. D 55, 299 (1997) hep-ph/9610445. 
[4] S. Capstick and W. Roberts, Prog. Part. Nucl. Phys. 45, S241 (2000) nucl-th/0008028.

[5] R. Bijker, E. Santopinto, Phys. Rev. C 80, 065210 (2009) arXiv:0912.4494 [nucl-th]].

[6] S. Capstick, A. Svarc, L. Tiator, J. Gegelia, M. M. Giannini, E. Santopinto, C. Hanhart and S. Scherer et al., Eur. Phys. J. A 35, 253 (2008) [arXiv:0711.1982 [hep-ph]].

[7] H. J. Lipkin, Phys. Rev. D 7, 846 (1973).

[8] R. Koniuk and N. Isgur, Phys. Rev. D 21, 1868 (1980) [Erratum-ibid. D 23, 818 (1981)].

[9] J. W. Darewych, M. Horbatsch and R. Koniuk, Phys. Rev. D 28, 1125 (1983).

[10] M. Warns, W. Pfeil and H. Rollnik, Phys. Lett. B 258, 431 (1991).

[11] R. K. Sahoo, A. R. Panda and A. Nath, Phys. Rev. D 52, 4099 (1995).

[12] G. Wagner, A. J. Buchmann and A. Faessler, Phys. Rev. C 58, 1745 (1998) nucl-th/9808005.

[13] R. Bijker, F. Iachello and A. Leviatan, Ann. Phys. 284, 89 (2000) nucl-th/0004034.

[14] E. Kaxiras, E. J. Moniz and M. Soyeur, Phys. Rev. D 32, 695 (1985).

[15] D. -H. Lu, A. W. Thomas and A. G. Williams, Phys. Rev. C 55, 3108 (1997) nucl-th/9612017.

[16] L. Yu, X. -L. Chen, W. -Z. Deng and S. -L. Zhu, Phys. Rev. D 73, 114001 (2006) hep-ph/0602171.

[17] N. Sharma, H. Dahiya, P. K. Chatley and M. Gupta, Phys. Rev. D 81, 073001 (2010) arXiv:1003.4338 [hep$\mathrm{ph}]$;

[18] C. L. Schat, C. Gobbi and N. N. Scoccola, Phys. Lett. B 356, 1 (1995) hep-ph/9506227.

[19] T. Haberichter, H. Reinhardt, N. N. Scoccola and H. Weigel, Nucl. Phys. A 615, 291 (1997) hep-ph/9610484.

[20] R. F. Lebed and R. H. TerBeek, Phys. Rev. D 83, 016009 (2011) arXiv:1011.3237 [hep-ph]].

[21] T. M. Aliev and A. Ozpineci, Nucl. Phys. B 732, 291 (2006) hep-ph/0406331.

[22] L. Wang and F. X. Lee, Phys. Rev. D 80, 034003 (2009) arXiv:0905.1944 [hep-ph]].

[23] M. N. Butler, M. J. Savage and R. P. Springer, Nucl. Phys. B 399, 69 (1993) hep-ph/9211247.

[24] D. B. Leinweber, T. Draper and R. M. Woloshyn, Phys. Rev. D 48, 2230 (1993) hep-lat/9212016.

[25] D. Arndt and B. C. Tiburzi, Phys. Rev. D 69, 014501 (2004) hep-lat/0309013.

[26] V. Pascalutsa, M. Vanderhaeghen and S. N. Yang, Phys. Rept. 437, 125 (2007) hep-ph/0609004.

[27] G. Ramalho, M. T. Peña and F. Gross, Eur. Phys. J. A 36, 329 (2008) arXiv:0803.3034 [hep-ph]].

[28] G. Ramalho, M. T. Peña and F. Gross, Phys. Rev. D 78, 114017 (2008) arXiv:0810.4126 [hep-ph]].

[29] G. Ramalho and M. T. Peña, J. Phys. G 36, 115011 (2009) arXiv:0812.0187 [hep-ph]].

[30] G. Ramalho and M. T. Peña, Phys. Rev. D 80, 013008 (2009) arXiv:0901.4310 [hep-ph]].

[31] K. Nakamura et al. [Particle Data Group], J. Phys. G 37, 075021 (2010).

[32] S. Taylor et al. [CLAS Collaboration], Phys. Rev. C
71, 054609 (2005) [Erratum-ibid. C 72, 039902 (2005)] hep-ex/0503014.

[33] D. Keller et al. [CLAS Collaboration], Phys. Rev. D 83, 072004 (2011) arXiv:1103.5701 [nucl-ex]].

[34] D. Keller et al. [CLAS Collaboration], Phys. Rev. D 85, 059903 (2012) arXiv:1111.5444 [nucl-ex]].

[35] G. Ramalho and K. Tsushima, Phys. Rev. D 87, 093011 (2013) arXiv:1302.6889 [hep-ph]].

[36] G. Ramalho, F. Gross, M. T. Peña and K. Tsushima, in Proceedings of the 4 th Workshop on Exclusive Reactions at High Momentum Transfer, edited by A. Radyushkin (World Scientific, Singapore, 2011), p. 287 arXiv:1008.0371 [hep-ph]].

[37] F. Gross, G. Ramalho and M. T. Peña, Phys. Rev. C 77, 015202 (2008) arXiv:nucl-th/0606029.

[38] F. Gross, G. Ramalho and M. T. Peña, Phys. Rev. D 85, 093005 (2012). arXiv:1201.6336 [hep-ph]].

[39] G. Ramalho and K. Tsushima, Phys. Rev. D 84, 054014 (2011) arXiv:1107.1791 [hep-ph]].

[40] G. Ramalho, K. Tsushima and F. Gross, Phys. Rev. D 80, 033004 (2009) arXiv:0907.1060 [hep-ph]].

[41] A. W. Thomas, Adv. Nucl. Phys. 13, 1 (1984).

[42] S. Theberge and A. W. Thomas, Nucl. Phys. A 393, 252 (1983).

[43] Y. Kohyama, K. Oikawa, K. Tsushima and K. Kubodera, Phys. Lett. B 186, 255 (1987); K. Tsushima, T. Yamaguchi, M. Takizawa, Y. Kohyama and K. Kubodera, Phys. Lett. B 205, 128 (1988); K. Tsushima, T. Yamaguchi, Y. Kohyama and K. Kubodera, Nucl. Phys. A 489, 557 (1988); T. Yamaguchi, K. Tsushima, Y. Kohyama and K. Kubodera, Nucl. Phys. A 500, 429 (1989).

[44] Y. Umino and F. Myhrer, Nucl. Phys. A 554, 593 (1993) nucl-th/9210018.

[45] G. Ramalho, K. Tsushima and A. W. Thomas, J. Phys. G 40, 015102 (2013) arXiv:1206.2207 [hep-ph]].

[46] G. Ramalho and K. Tsushima, Phys. Rev. D 82, 073007 (2010) arXiv:1008.3822 [hep-ph]].

[47] G. Ramalho and K. Tsushima, Phys. Rev. D 86, 114030 (2012) arXiv:1210.7465 [hep-ph]].

[48] C. Amsler et al. [Particle Data Group], Phys. Lett. B 667, 1 (2008). [See page 1023 for information about the Naive Quark Model].

[49] I. C. Cloet, D. B. Leinweber and A. W. Thomas, Phys. Rev. C 65, 062201 (2002) hep-ph/0203023.

[50] B. Julia-Diaz, T. S. Lee, T. Sato and L. C. Smith, Phys. Rev. C 75, 015205 (2007).

[51] E. E. Jenkins and A. V. Manohar, Phys. Lett. B 255, 558 (1991).

[52] V. Bernard, N. Kaiser, J. Kambor and U. G. Meissner, Nucl. Phys. B 388, 315 (1992).

[53] V. Bernard, Prog. Part. Nucl. Phys. 60, 82 (2008) arXiv:0706.0312 [hep-ph]].

[54] L. Tiator, D. Drechsel, O. Hanstein, S. S. Kamalov and S. N. Yang, Nucl. Phys. A 689, 205 (2001) nucl-th/0012046.

[55] V. V. Molchanov et al. [SELEX Collaboration], Phys. Lett. B 590, 161 (2004) hep-ex/0402026. 